\title{
Comparing different thrombolytic dosing regimens for treatment of acute pulmonary embolism
}

\author{
Ammar Ghanem* and Sachin Yende \\ University of Pittsburgh Department of Critical Care Medicine: Evidence-Based Medicine Journal Club, edited by Sachin Yende
}

\section{Expanded abstract Citation}

Ch en Wang, Zh enguo Zhai, Yuanhua Yang, Qi Wu, Zhaozhong Cheng, Lirong Liang, Huaping Dai, Kewu Huang, Weixuan Lu, Zhonghe Zhang, Xiansheng Cheng, Ying H Shen, For the China Venous Thromboembolism (VTE) Study Group: Chest 2010, 137:254-262. Trial registration: clinicaltrials.gov; Identifier: NCT00781378.

\section{Background}

Optimal dosing of recombinant tissue-type plasminogen activator (rt-PA) is important in treating pulmonary thromboembolism (PTE).

\section{Methods \\ Objective: The aim of this study was to compare the efficacy and safety of a $50 \mathrm{mg} / 2 \mathrm{~h}$ rt-PA regimen with a $100 \mathrm{mg} / 2 \mathrm{~h}$ rt-PA regimen in patients with acute PTE. Design: A prospective, randomized, open label trial. \\ Setting: A multicenter trial in China. \\ Subjects: 118 patients with acute PTE and either hemo- dynamic instability or massive pulmonary artery obstruction. \\ Intervention: Patients were randomly assigned to receive a treatment regimen of either $\mathrm{rt}-\mathrm{PA}$ at $50 \mathrm{mg} / 2 \mathrm{~h}(\mathrm{n}=65)$ or $100 \mathrm{mg} / 2 \mathrm{~h}(\mathrm{n}=53)$. \\ Outcomes: The efficacy was determined by observing the improvements of right ventricular dysfunctions (RVDs) on echocardiograms, lung perfusion defects on ventila- tion perfusion lung scans, and pulmonary artery obstruc- tions on CT angiograms. The adverse events, including death, bleeding, and PTE recurrence, was also evaluated.}

*Correspondence: ymghanem@hotmail.com

Department of Critical Care Medicine, University of Pittsburgh School of Medicine, Pittsburgh, Pennsylvania, USA

\section{Results}

Progressive improvements in RVDs, lung perfusion defects, and pulmonary artery obstructions were found to be similar in both treatment groups. This is true for patients with either hemodynamic instability or massive pulmonary artery obstruction. Three (6\%) patients in the rt-PA $100 \mathrm{mg} / 2 \mathrm{~h}$ group and one (2\%) in the rt-PA $50 \mathrm{mg} / 2 \mathrm{~h}$ group died as the result of either PTE or bleeding. Importantly, the $50 \mathrm{mg} / 2 \mathrm{~h}$ rt-PA regimen resulted in less bleeding tendency than the $100 \mathrm{mg} / 2 \mathrm{~h}$ regimen ( $3 \%$ vs. $10 \%$ ), especially in patients with a body weight, $65 \mathrm{~kg}(14.8 \%$ vs. $41.2 \%, P=0.049)$. No fatal recurrent PTE was found in either group.

\section{Conclusions}

Compared with the $100 \mathrm{mg} / 2 \mathrm{~h}$ regimen, the $50 \mathrm{mg} / 2 \mathrm{~h}$ rt-PA regimen exhibits similar efficacy and perhaps better safety in patients with acute PTE. These findings support the notion that optimizing rt-PA dosing is worthwhile when treating patients with PTE.

\section{Commentary}

Acute pulmonary thromboembolism (PTE) is a disease with variable clinical severity that can range from no symptoms to severe hypoxia, hypotension, right heart failure and death. Thrombolytic therapy is known to improve physiologic parameters and right heart function in PTE. This therapy is routinely used in patients who have hemodynamic instability. However, its role in patients with large PTE in the absence of hemodynamic instability, particularly in the subset with right ventricular strain, is controversial. Few large randomized clinical trials(RCTs) have been conducted to assess efficacy of thrombolytic therapy for different subgroups of patients with PTE and to compare different dosing regimens, Current recommendations are largely based on results of observational studies or meta-analyses of small RCT [1-3].

Recombinant tissue-type plasminogen activator (rt-PA) is currently the most commonly used thrombolytic therapy for PTE. Similar to most thrombolytic agents, 
rt-PA carries a significant dose-dependent risk of bleeding, and it is the most common adverse effect associated with thrombolytic therapy for PTE. In a retrospective analysis of 104 patients with PTE who receive rt-PA, 20 patients (19\%) had major bleeding [4]. The most devastating complication is intracranial bleed and it occurs in up to $3 \%$ of patients. Thus, optimal dosing to maximize benefits and minimize bleeding complications is important.

Few studies had compared different thrombolytic doses for PTE [5,6]. For example, Goldhaber and colleagues compared $0.6 \mathrm{mg} / \mathrm{kg}$ over $15 \mathrm{~min}$ (maximum dose of $50 \mathrm{mg}$ ) and $100 \mathrm{mg}$ over 2 hours in 90 patients. No significant differences were detected between both groups with regards to bleeding complications and efficacy, as measured by perfusion lung scans, pulmonary angiograms and echocardiograms.

With this background, Wang and colleagues conducted a randomized, multicenter study to compare low vs. high dose rt-PA in treatment of acute massive PTE [7]. Patients were included if they were 18 years or older and present with symptoms of acute PTE within 15 days of enrolment. This study enrolled patients with large PTE, as evidence by hemodynamic instability (systolic blood pressure $[\mathrm{BP}]<90 \mathrm{mmHg}$ or drop in systolic BP of at least $40 \mathrm{mmHg}$ for at least 15 minutes), cardiogenic shock or those with anatomically massive PTE (CT scan showed occlusion of more than 2 lobar arteries, or V/Q scan showed occlusion $>7$ segments combined with right ventricular dysfunction on echocardiography). For sample size calculation, the authors calculated the number of patient needed to demonstrate a reduction in the $\mathrm{CT}$ obstruction score by 10 points. A total number of 118 patients were enrolled (65 in the low dose and 53 in the high dose group). The primary endpoints were efficacy of thrombolysis, as measured by improvement in right ventricular function by echocardiogram, improvement in perfusion by V/Q scan and improvement in CT obstruction score. No differences in efficacy were observed between the two groups. Secondary endpoints were safety endpoints, including bleeding risk. Bleeding events were categorized into major and minor events. Major events included bleeding leading to death, caused a drop in hemoglobin $>2 \mathrm{~g} / \mathrm{dl}$, or required transfusion more than $400 \mathrm{cc}$ blood and intra cranial hemorrhage. Minor events included bleeding that led to a drop of less than $2 \mathrm{~g} / \mathrm{dl}$ in hemoglobin. Other secondary endpoints were recurrence of PTE and death. Although bleeding risk was no different, in subgroup analysis stratified by body weight, the risk of total number of bleeding episodes were less with the low dose regimen than in the high dose regimen, especially in patients with body weight $<65 \mathrm{~kg}$ (14.8\% in the low dose vs. $41.2 \%$ in the high dose group, $P=0.049$ ) or $\mathrm{BMI}<24(8.7 \%$ in the low dose vs. $42.9 \%$ in the high dose group, $P=0.014)$.
To date, this study is the largest one to compare different dosing regimens of rt-PA for acute PTE. The subgroup analysis according to body weight suggests that using weight-based dosing may reduce bleeding complications. However the study has limitations. The authors chose a surrogate endpoint and its clinical significance is unclear. For example, it is difficult to estimate the clinical significance of a 1 point decrease in the CT obstruction score. Although mortality would be an important outcome measure, it was a secondary outcome in this study and there were only a few deaths.

This study highlights the importance of considering alternative study designs to compare different dose regimens of thrombolytic therapy for PTE. For instance, lower dose may have similar efficacy but lower bleeding complications, thus such studies should be designed as non-inferiority or equivalence trial, with the hypothesis that clinical efficacy would be similar but bleeding risk would be lower.

In conclusion, this trial did not prove differences in efficacy between low dose and high dose rt-PA regimens. The secondary analyses showing that lower dose of rt-PA may lower the risk of bleeding suggest a need for additional studies to use weight-based regimens to reduce risk of bleeding.

Competing interests

The authors declare that they have no competing interests.

Published: 15 October 2010

\section{References}

1. Capstick T, Henry MT: Efficacy of thrombolytic agents in the treatment of pulmonary embolism. Eur Respir J 2005, 26:864-874.

2. Agnelli $G$, Becattini $C$, Kirschstein T: Thrombolysis vs heparin in the treatment of pulmonary embolism: a clinical outcome-based metaanalysis. Arch Intern Med 2002, 162:2537-2541.

3. Wan S, Quinlan DJ, Agnelli G, Eikelboom JW: Thrombolysis compared with heparin for the initial treatment of pulmonary embolism: a meta-analysis of the randomized controlled trials. Circulation 2004, 110:744-749.

4. Fiumara K, Kucher N, Fanikos J, Goldhaber SZ: Predictors of major hemorrhage following fibrinolysis for acute pulmonary embolism. Am J Cardiol 2006, 97:127-129.

5. Goldhaber SZ, Agnelli G, Levine MN: Reduced dose bolus alteplase vs conventional alteplase infusion for pulmonary embolism thrombolysis. An international multicenter randomized trial. The Bolus Alteplase Pulmonary Embolism Group. Chest 1994, 106:718-724.

6. Levine M, Hirsh J, Weitz J, Cruickshank M, Neemeh J, Turpie AG, Gent M: A randomized trial of a single bolus dosage regimen of recombinant tissue plasminogen activator in patients with acute pulmonary embolism. Chest 1990, 98:1473-1479.

7. Wang C, Zhai Z, Yang Y, Wu Q, Cheng Z, Liang L, Dai H, Huang K, Lu W, Zhang $Z$, Cheng X, Shen YH: Efficacy and safety of low dose recombinant tissuetype plasminogen activator for the treatment of acute pulmonary thromboembolism: a randomized, multicenter, controlled trial. Chest 2010, $137: 254-262$.

doi:10.1186/cc9287

Cite this article as: Ghanem A, Yende S: Comparing different thrombolytic dosing regimens for treatment of acute pulmonary embolism. Critical Care 2010, 14:323. 